\title{
BMJ
}

\section{Effect of fibre, antispasmodics, and peppermint oil in the treatment of irritable bowel syndrome: systematic review and meta-analysis}

\author{
Alexander C Ford, clinical fellow, ${ }^{1}$ Nicholas I Talley, professor of medicine, ${ }^{2}$ Brennan M R Spiegel, assistant \\ professor of medicine, ${ }^{3}$ Amy E Foxx-Orenstein, associate professor of medicine, ${ }^{4}$ Lawrence Schiller, clinical \\ professor, ${ }^{5}$ Eamonn M M Quigley, professor of medicine and human physiology, ${ }^{6}$ Paul Moayyedi, professor \\ of gastroenterology ${ }^{1}$
}

${ }^{1}$ Gastroenterology Division, McMaster University, Health Sciences Centre, 1200 Main Street West, Hamilton, ON, L8N 3Z5, Canada

${ }^{2}$ Department of Medicine, Mayo Clinic Florida, Jacksonville, FL, USA

${ }^{3}$ VA Greater Los Angeles

Healthcare System; UCLA/VA

Center for Outcomes Research

and Education, Los Angeles, CA,

USA

${ }^{4}$ Division of Gastroenterology and Hepatology, Mayo Clinic

Rochester, MN, USA

${ }^{5}$ Digestive Health Associates of Texas, Baylor University Medical Center, Dallas, TX, USA

${ }^{6}$ Department of Medicine, Cork University Hospital, Ireland

Correspondence to: A C Ford alexf12399@yahoo.com

Cite this as: BMJ 2008;337:a2313 doi:10.1136/bmi.a2313

\section{ABSTRACT}

Objective To determine the effect of fibre, antispasmodics, and peppermint oil in the treatment of irritable bowel syndrome.

Design Systematic review and meta-analysis of randomised controlled trials.

Data sources Medline, Embase, and the Cochrane controlled trials register up to April 2008.

Review methods Randomised controlled trials comparing fibre, antispasmodics, and peppermint oil with placebo or no treatment in adults with irritable bowel syndrome were eligible for inclusion. The minimum duration of therapy considered was one week, and studies had to report either a global assessment of cure or improvement in symptoms, or cure of or improvement in abdominal pain, after treatment. A random effects model was used to pool data on symptoms, and the effect of therapy compared with placebo or no treatment was reported as the relative risk ( $95 \%$ confidence interval) of symptoms persisting.

Results 12 studies compared fibre with placebo or no treatment in 591 patients (relative risk of persistent symptoms $0.87,95 \%$ confidence interval 0.76 to 1.00$)$. This effect was limited to ispaghula $(0.78,0.63$ to 0.96$)$. Twenty two trials compared antispasmodics with placebo in 1778 patients $(0.68,0.57$ to 0.81$)$. Various antispasmodics were studied, but otilonium (four trials, 435 patients, relative risk of persistent symptoms 0.55 , 0.31 to 0.97 ) and hyoscine (three trials, 426 patients, $0.63,0.51$ to 0.78 ) showed consistent evidence of efficacy. Four trials compared peppermint oil with placebo in 392 patients $(0.43,0.32$ to 0.59$)$.

Conclusion Fibre, antispasmodics, and peppermint oil were all more effective than placebo in the treatment of irritable bowel syndrome.

\section{INTRODUCTION}

Irritable bowel syndrome is a functional gastrointestinal disorder characterised by abdominal pain or discomfort and accompanied by a change in bowel habit. ${ }^{1}$ The condition has a population prevalence of between $5 \%$ and $20 \%$ in community surveys. ${ }^{2-4}$ No known structural or anatomical explanation accounts for the pathophysiology of irritable bowel syndrome, and the exact cause remains unknown, although several mechanisms have been proposed. Altered gastrointestinal motility may contribute to the change in bowel habit reported by some patients, ${ }^{5}$ and a combination of smooth muscle spasm, visceral hypersensitivity, and abnormalities of central pain processing may explain the abdominal pain that is an essential part of the symptom complex. ${ }^{67}$

Irritable bowel syndrome is a chronic relapsing and remitting condition, ${ }^{89}$ and a significant proportion of patients will consult their general practitioner with symptoms. ${ }^{910}$ Current guidelines for the management of irritable bowel syndrome in the United Kingdom recommend that the diagnosis should be made on clinical grounds alone, without the need for invasive investigations, unless alarm symptoms such as rectal bleeding or weight loss are present. ${ }^{112}$ As a result general practitioners are increasingly responsible for the initial management of patients with irritable bowel syndrome and are expected to refer only a minority to secondary care.

If they are to fulfil this role effectively, general practitioners need efficacious treatments that do not require monitoring and are cheap, safe, and readily available. This is particularly relevant at the present time as newer and more expensive drugs have either failed to show efficacy or been withdrawn from the market owing to concerns about serious adverse events. Traditionally, people with irritable bowel syndrome were instructed to increase their daily intake of dietary fibre, because of its potentially beneficial effects on intestinal transit time. ${ }^{13}$ When this failed, various types of smooth muscle relaxants and antispasmodics were used in an attempt to ameliorate symptoms, particularly pain and bloating. ${ }^{12}$ More recently, peppermint oil, which has been shown to have antispasmodic properties, ${ }^{14}$ has been available over the counter and has been used in the treatment of irritable bowel syndrome. 
Whether any of these agents are effective in the treatment of irritable bowel syndrome is controversial. Results of randomised controlled trials are conflicting, and many have been underpowered to detect a difference between active treatment and control intervention. Systematic reviews have also come to different conclusions about the efficacy of the three treatments in irritable bowel syndrome. ${ }^{15-22}$ As a result confusion exists as to the roles of these agents, with current management guidelines for irritable bowel syndrome making varying recommendations. ${ }^{11} 122324$

We carried out a systematic review and metaanalysis to determine the effect of fibre, antispasmodics, and peppermint oil in the treatment of irritable bowel syndrome.

\section{METHODS}

We searched the medical literature using Medline (1950 to April 2008), Embase (1980 to April 2008), and the Cochrane controlled trials register (2007). We considered randomised controlled trials of adults ( $>16$ years) with a diagnosis of irritable bowel syndrome based on a clinician's opinion or that met specific diagnostic criteria (Manning, Kruis score, Rome I, II, or III), combined with the results of investigations to exclude organic disease if trial investigators thought this necessary. The studies had to compare fibre, antispasmodics, and peppermint oil with placebo or no treatment. Participants were required to be followedup for at least one week, and studies had to report either a global assessment of cure or improvement of symptoms, or cure or improvement of abdominal pain, after treatment. This was preferably as reported by the patient, but could be documented by a doctor. If studies included patients with other functional gastrointestinal disorders, then we excluded these patients from our analyses if trial reporting allowed this, but if this was not possible we excluded the studies from the meta-analysis. We also considered as eligible for inclusion the first period of cross over randomised controlled trials. To allow steady state plasma concentrations of the agents to be achieved we considered one week as the minimum duration of treatment.

We identified studies on irritable bowel syndrome using the terms "irritable bowel syndrome" and "functional diseases, colon" (both as medical subject heading and free text terms), and "IBS, spastic colon, irritable colon", and "functional adj5 bowel" (as free text terms). These were combined using the set operator AND with studies identified with the terms: "dietary fibre", "cereals", "psyllium", "sterculia", "karaya gum", "parasympatholytics", "scopolamine”, "trimebutine", "muscarinic antagonists", "butylscopolammonium bromide" (both as medical subject headings and free text terms), and the following free text terms: "bulking agent", "psyllium fibre", "fibre", "husk", "bran", "ispaghula", "wheat bran", "spasmolytics", "spasmolytic agents", "antispasmodics", "mebeverine", "alverine", "pinaverium bromide", "otilonium bromide", "cimetropium bromide",

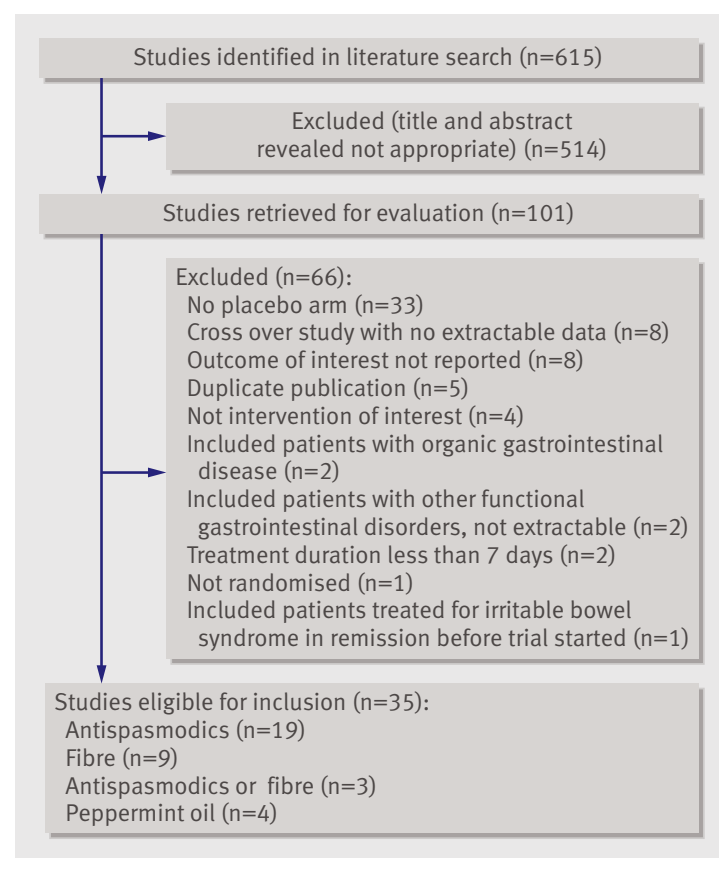

Fig 1 | Flow diagram of studies in systematic review

"hyoscine butyl bromide", "butylscopolamine", "peppermint oil", and "colpermin".

No language restrictions were applied. The lead reviewer evaluated the abstracts of papers identified by the initial search for appropriateness to the study question. Potentially relevant papers were obtained and evaluated in detail. Foreign language papers were translated when required. We hand searched abstract books of conference proceedings between 2001 and 2007 to identify potentially eligible studies. The reference lists of all identified relevant studies were used to carry out a recursive search of the literature. Two reviewers independently assessed articles using predesigned eligibility forms, according to eligibility criteria defined prospectively. Any disagreement between investigators was resolved by consensus.

\section{Outcome assessment}

The primary outcomes assessed were the efficacy of fibre, antispasmodics, and peppermint oil compared with placebo or no treatment on global symptoms of irritable bowel syndrome or abdominal pain after treatment. Secondary outcomes included efficacy according to specific type of fibre or antispasmodic, and adverse events as a result of treatment.

\section{Data extraction}

Two reviewers independently extracted data on to an Excel spreadsheet (XP professional; Microsoft, Redmond, WA) as dichotomous outcomes (persistent or unimproved global symptoms of irritable bowel syndrome, or persistent or unimproved abdominal pain). In addition we extracted the following clinical data for each trial: setting (primary, secondary, or tertiary care), number of centres, country, dose and duration of treatment, total number of adverse events 
reported, definition of irritable bowel syndrome used, primary outcome measure used to define improvement in symptoms or cure after treatment, method of generation of the randomisation schedule, method for allocation concealment, level of blinding, proportion of female patients, subtype of irritable bowel syndrome according to predominant stool pattern, and duration of follow-up. Data were extracted as intention to treat analyses where all dropouts are assumed to be treatment failures, whenever this was allowed by trial reporting. If this was not clear from the original article then we carried out an analysis on all patients with reported evaluable data.

\section{Study quality}

Two reviewers independently assessed study quality according to the Jadad scale. ${ }^{25}$ This records whether a study is described as randomised and double blind, the methods for generation of the allocation schedule and double blinding, and whether there is a description of dropouts during the trial.

\section{Data synthesis and statistical analysis}

We pooled data using a random effects model to give a more conservative estimate of the effect of individual treatments, allowing for any heterogeneity between studies. ${ }^{26}$ The effects of different interventions were expressed as a relative risk ( $95 \%$ confidence interval) of global symptoms of irritable bowel syndrome or abdominal pain persisting with fibre, antispasmodics, or peppermint oil compared with placebo or no treatment. For rare outcomes, such as adverse events, when no patients in one or both treatment arms had the outcome of interest in a single study, we added 0.5 to all four cells for the purposes of the analysis. From the reciprocal of the risk difference from the meta-analysis we calculated the number needed to treat and $95 \%$ confidence intervals. We used the $\mathrm{I}^{2}$ statistic, with a cutoff point of $25 \%,{ }^{27}$ to assess heterogeneity between studies and the $\chi^{2}$ test with a $\mathrm{P}$ value $<0.10$ to define a significant degree of heterogeneity. We planned to do sensitivity analyses a priori according to type of fibre or antispasmodic, predominant stool pattern of patients, and study quality according to the Jadad scale. If adverse events were statistically significantly increased with active treatment we calculated the number needed to harm and a 95\% confidence interval using the formula: number needed to harm $=1 /(1$-relative risk) $\times$ control adverse event rate.

We used Review Manager version 4.2.8 (Nordic Cochrane Centre, Copenhagen, Denmark) and StatsDirect version 2.4.4 (Sale, Cheshire, England) to generate forest plots of pooled relative risks and risk differences for primary and secondary outcomes with $95 \%$ confidence intervals. We used the Egger and Begg tests to assess funnel plots for evidence of publication bias. ${ }^{28}$

\section{RESULTS}

The search strategy generated 615 citations, of which 101 were potentially relevant and retrieved for assessment (fig 1). Of these, 66 were excluded for various reasons, leaving 35 eligible randomised

\begin{tabular}{|c|c|c|c|c|c|c|c|c|}
\hline Study & Country & Setting & $\begin{array}{l}\text { Diagnostic criteria for } \\
\text { irritable bowel syndrome }\end{array}$ & $\begin{array}{l}\text { Criteria to define symptom } \\
\text { improvement after therapy }\end{array}$ & $\begin{array}{l}\text { Sample } \\
\text { size }\end{array}$ & Fibre type & $\begin{array}{c}\text { Duration of } \\
\text { therapy }\end{array}$ & Jadad score \\
\hline Soltoft $1976^{\text {w2 }}$ & Denmark & Tertiary care & $\begin{array}{l}\text { Clinical diagnosis and } \\
\text { investigations }\end{array}$ & $\begin{array}{l}\text { Patient reported improvement in } \\
\text { global symptoms }\end{array}$ & 59 & $\begin{array}{l}\text { Miller's bran } 30 \text { g per } \\
\text { day }\end{array}$ & 6 weeks & 4 \\
\hline $\begin{array}{l}\text { Manning } \\
1977^{\mathrm{w} 3}\end{array}$ & England & Tertiary care & $\begin{array}{l}\text { Clinical diagnosis and } \\
\text { investigations }\end{array}$ & $\begin{array}{l}\text { Any decrease in patient reported } \\
\text { percentage of days with pain }\end{array}$ & 26 & $\begin{array}{l}\text { Bran } 20 \text { g per day } \\
\text { (wheat bran or } \\
\text { whole wheat bread) }\end{array}$ & 6 weeks & 3 \\
\hline Ritchie $1979^{\text {w33 }}$ & England & Tertiary care & $\begin{array}{l}\text { Clinical diagnosis and } \\
\text { investigations }\end{array}$ & $\begin{array}{l}\text { Patient reported improvement in } \\
\text { global symptoms (doctor required } \\
\text { to agree) }\end{array}$ & 24 & $\begin{array}{l}\text { Ispaghula husk two } \\
\text { sachets per day }\end{array}$ & 3 months & 4 \\
\hline $\begin{array}{l}\text { Longstreth } \\
1981^{\text {w9 }}\end{array}$ & USA & $\begin{array}{l}\text { Secondary } \\
\text { care }\end{array}$ & Clinical diagnosis & $\begin{array}{l}\text { Patient reported improvement in } \\
\text { global symptoms }\end{array}$ & 77 & $\begin{array}{l}\text { Metamucil } 6.4 \mathrm{~g} \text { per } \\
\text { day }\end{array}$ & 8 weeks & 4 \\
\hline Arthurs $1983^{\text {w8 }}$ & Ireland & $\begin{array}{l}\text { Secondary } \\
\text { care }\end{array}$ & Clinical diagnosis & $\begin{array}{l}\text { Doctor reported improvement in } \\
\text { global symptoms }\end{array}$ & 78 & $\begin{array}{l}\text { Ispaghula husk two } \\
\text { sachets per day }\end{array}$ & 4 weeks & 4 \\
\hline Nigam $1984^{\text {w35 }}$ & India & $\begin{array}{l}\text { Secondary } \\
\text { care }\end{array}$ & $\begin{array}{l}\text { Clinical diagnosis and } \\
\text { investigations }\end{array}$ & $\begin{array}{l}\text { Patient reported improvement in } \\
\text { global symptoms (doctor required } \\
\text { to agree) }\end{array}$ & 42 & $\begin{array}{l}\text { Ispaghula husk } \\
\text { (dose unclear) }\end{array}$ & 12 weeks & 3 \\
\hline Kruis $1986^{\text {w34 }}$ & Germany & Tertiary care & $\begin{array}{l}\text { Clinical diagnosis and } \\
\text { investigations }\end{array}$ & $\begin{array}{l}\text { Patient reported improvement in } \\
\text { global symptoms }\end{array}$ & 80 & $\begin{array}{l}\text { Wheat bran } 15 \mathrm{~g} \text { per } \\
\text { day }\end{array}$ & 16 weeks & 2 \\
\hline Lucey $1987^{w 7}$ & England & Tertiary care & $\begin{array}{l}\text { Manning criteria and } \\
\text { investigations }\end{array}$ & $\begin{array}{l}\text { Any decrease in global symptom } \\
\text { score }\end{array}$ & 28 & $\begin{array}{l}\text { Wheat bran biscuits } \\
15.6 \text { g per day }\end{array}$ & 3 months & 3 \\
\hline Prior $1987^{\text {w6 }}$ & England & Tertiary care & $\begin{array}{l}\text { Clinical diagnosis and } \\
\text { investigations }\end{array}$ & $\begin{array}{l}\text { Patient reported absence of } \\
\text { abdominal pain }\end{array}$ & 80 & $\begin{array}{l}\text { Ispaghula husk } \\
\text { three sachets per } \\
\text { day }\end{array}$ & 12 weeks & 4 \\
\hline Jalihal $1990^{\text {w5 }}$ & India & $\begin{array}{l}\text { Secondary } \\
\text { care }\end{array}$ & $\begin{array}{l}\text { Clinical diagnosis and } \\
\text { investigations }\end{array}$ & $\begin{array}{l}\text { Patient reported improvement in } \\
\text { global symptoms }\end{array}$ & 20 & $\begin{array}{l}\text { Ispaghula husk } 30 \mathrm{~g} \\
\text { per day }\end{array}$ & 4 weeks & 4 \\
\hline Fowlie $1992^{\text {w4 }}$ & Scotland & Tertiary care & $\begin{array}{l}\text { Clinical diagnosis and } \\
\text { investigations }\end{array}$ & $\begin{array}{l}\text { Patient reported improvement in } \\
\text { global symptoms }\end{array}$ & 49 & $\begin{array}{l}4.1 \mathrm{~g} \text { of unspecified } \\
\text { fibre per day }\end{array}$ & 12 weeks & 4 \\
\hline Rees $2005^{\mathrm{w} 1}$ & England & Tertiary care & $\begin{array}{l}\text { Rome I criteria and } \\
\text { investigations }\end{array}$ & $\begin{array}{l}\text { Patient reported improvement in } \\
\text { global symptoms }\end{array}$ & 28 & $\begin{array}{l}\text { Wheat bran } 10 \text { to } \\
20 \text { g per day }\end{array}$ & 12 weeks & 1 \\
\hline
\end{tabular}


controlled trials; nine compared fibre with placebo or no treatment, ${ }^{\text {w1-w } 9} 19$ compared antispasmodics with placebo, ${ }^{\text {w10-w28 }}$ four compared peppermint oil with placebo, ${ }^{\text {w29-w32 }}$ and three compared both fibre and antispasmodics with placebo. ${ }^{\text {w33-w35 }}$

Fibre

Twelve trials compared fibre with placebo or, in one instance, a low fibre diet, ${ }^{\mathrm{w} 3}$ totalling 591 patients with irritable bowel syndrome. ${ }^{\text {w1-w9 }}$ w3-w35 The proportion of women in the trials ranged between $20 \%$ and $90 \%$. Only three studies reported on subtype of irritable bowel syndrome according to predominant stool pattern. ${ }^{\mathrm{w} 1 \mathrm{w} 4 \mathrm{w} 6}$ Two recruited only patients with predominant constipation, ${ }^{\mathrm{w} 1 \mathrm{w} 4}$ and in the other trial $49 \%$ of patients had predominant constipation. ${ }^{\text {w6 }}$ Table 1 shows the characteristics of the studies. Five studies used bran, ${ }^{\text {w1-w3 w7 w34 }}$ six used ispaghula husk, ${ }^{\text {w5 w6 w8 w9 }}$ w33 w35 and one used "concentrated" fibre of an unspecified type. ${ }^{\mathrm{w} 4}$ Seven of the studies scored 4 or

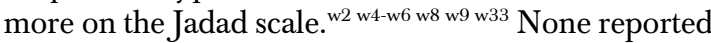
the method of allocation concealment.
Overall, 155 of $300(52 \%)$ patients assigned to fibre had persistent or unimproved symptoms after treatment compared with 168 of 291 (57\%) allocated to placebo or a low fibre diet (relative risk 0.87, 95\% confidence interval 0.76 to $1.00, \mathrm{P}=0.05$ ), with no statistically significant heterogeneity detected between studies $\left(\mathrm{I}^{2}=14.2 \%, \mathrm{P}=0.31\right.$; fig 2$)$. The number needed to treat with fibre to prevent one patient with persistent symptoms was 11 (95\% confidence interval 5 to 100). The funnel plot showed no statistically significant asymmetry (Egger test, $\mathrm{P}=0.84$ ), suggesting no evidence of publication bias. When only the seven studies scoring 4 or more on the Jadad scale were considered in the analysis the treatment effect for fibre was no longer statistically significant (relative risk of persistent symptoms $0.90,0.75$ to 1.08$).{ }^{\text {w2 }}$ w4-w6 w8 w9 w33

\section{Bran}

Five studies compared bran with placebo or a low fibre diet in a total of 221 patients. ${ }^{\mathrm{wl}-\mathrm{w} 3}$ w7 w34 Only one study scored 4 or more on the Jadad scale. ${ }^{\text {w2 }}$ Sixty two of 114 $(54 \%)$ patients assigned to bran had persistent

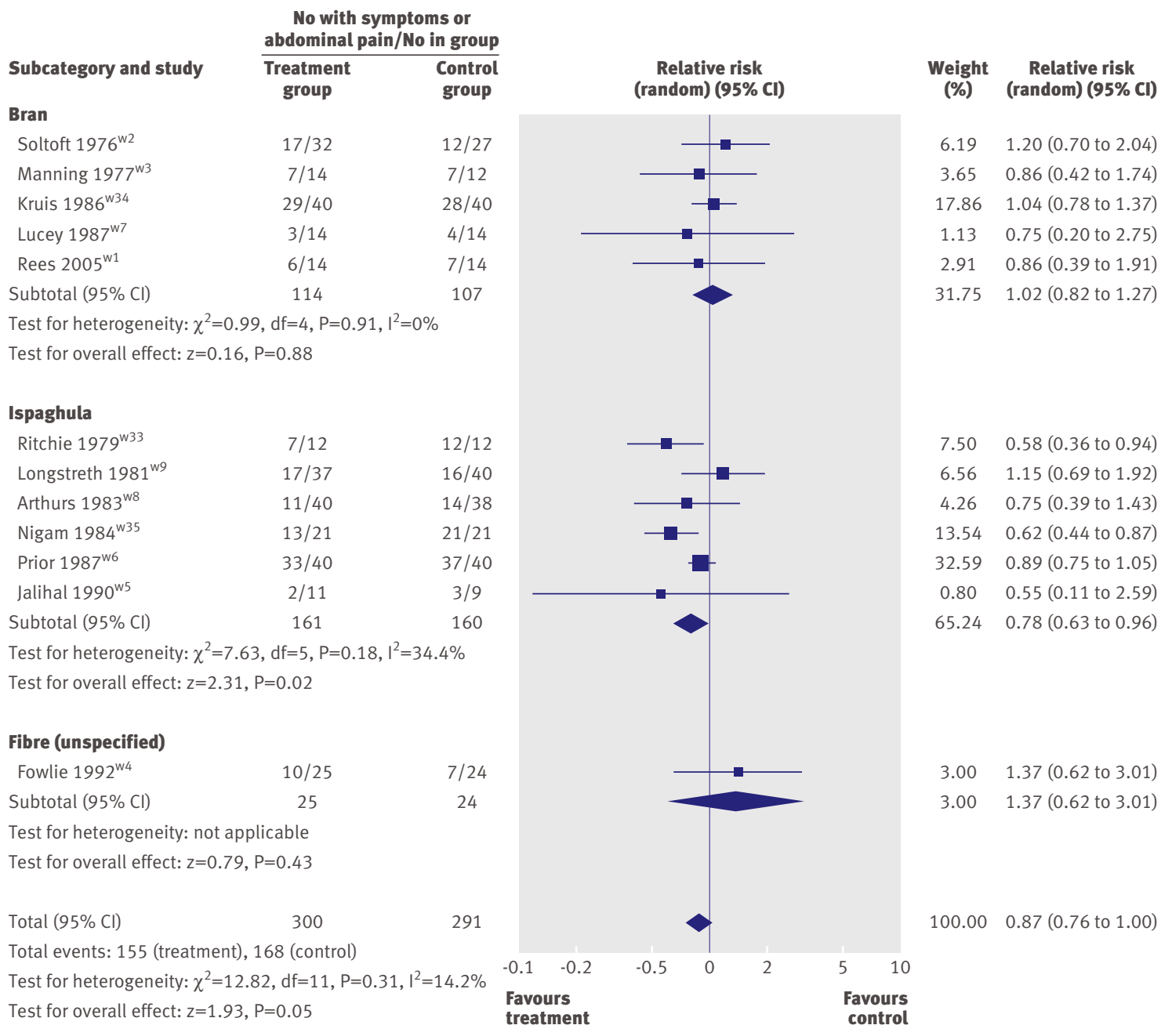

Fig 2 | Forest plot of randomised controlled trials of fibre versus placebo or low fibre diet in irritable bowel syndrome. Events are number of patients with either global symptoms of irritable bowel syndrome or abdominal pain unimproved or persistent after treatment 
symptoms after treatment compared with 58 of 107 (54\%) allocated to placebo or a low fibre diet. Bran had no significant effect on irritable bowel syndrome (relative risk of persistent or unimproved symptoms $1.02,0.82$ to 1.27 ; fig 2 ), with no statistically significant heterogeneity detected between studies $\left(I^{2}=0 \%\right.$, $\mathrm{P}=0.91$ ), and no evidence of funnel plot asymmetry (Egger test, $\mathrm{P}=0.28$ ).

\section{Ispaghula}

Six studies randomised 321 patients to ispaghula or placebo. ${ }^{\text {w5 w6 w8 w9 w33 w35 Eighty three of } 161(52 \%)}$ patients allocated to ispaghula had persistent symptoms after treatment compared with 103 of 160 $(64 \%)$ receiving placebo. Ispaghula was effective in treating irritable bowel syndrome (relative risk of persistent or unimproved symptoms $0.78,0.63$ to 0.96 ; fig 2), with statistically significant heterogeneity detected between studies $\left(\mathrm{I}^{2}=34.4 \%, \mathrm{P}=0.18\right)$. The number needed to treat with ispaghula to prevent one patient having persistent symptoms was 6 (3 to 50). No evidence of funnel plot asymmetry was found (Egger test, $\mathrm{P}=0.43$ ). Five of the six studies using ispaghula scored 4 or more on the Jadad scale. ${ }^{\text {w5 }}$ w6 w8 w9 w33 When only these studies were considered in the analysis the treatment effect for ispaghula was no longer statistically

Table 2 | Characteristics of randomised controlled trials of antispasmodics versus placebo or no treatment in irritable bowel syndrome

\begin{tabular}{|c|c|c|c|c|c|c|c|c|}
\hline Study & Country & Setting & $\begin{array}{l}\text { Diagnostic criteria } \\
\text { for irritable bowel } \\
\text { syndrome }\end{array}$ & $\begin{array}{l}\text { Criteria to define symptom } \\
\text { improvement after therapy }\end{array}$ & $\begin{array}{l}\text { Sample } \\
\text { size }\end{array}$ & Antispasmodic and dose & $\begin{array}{l}\text { Duration } \\
\text { of therapy }\end{array}$ & $\begin{array}{l}\text { Jadad } \\
\text { score }\end{array}$ \\
\hline Levy $1977^{w 22}$ & France & Secondary care & $\begin{array}{l}\text { Clinical diagnosis } \\
\text { and investigations }\end{array}$ & $\begin{array}{l}\text { Improvement in global symptoms } \\
\text { (unclear whether patient reported) }\end{array}$ & 50 & $\begin{array}{l}\text { Pinaverium } 50 \mathrm{mg} \text { three } \\
\text { times daily }\end{array}$ & 15 days & 3 \\
\hline Moshal $1979^{\text {w15 }}$ & $\begin{array}{l}\text { South } \\
\text { Africa }\end{array}$ & Tertiary care & $\begin{array}{l}\text { Clinical diagnosis } \\
\text { and investigations }\end{array}$ & $\begin{array}{l}\text { Patient reported improvement in } \\
\text { abdominal pain }\end{array}$ & 20 & $\begin{array}{l}\text { Trimebutine } 200 \mathrm{mg} \text { three } \\
\text { times daily }\end{array}$ & 4 weeks & 4 \\
\hline Piai $1979^{\text {w19 }}$ & Italy & Tertiary care & $\begin{array}{l}\text { Clinical diagnosis } \\
\text { and investigations }\end{array}$ & $\begin{array}{l}\text { Physician reported improvement in global } \\
\text { symptoms }\end{array}$ & 18 & $\begin{array}{l}\text { Prifinium bromide } 30 \mathrm{mg} \\
\text { three times daily }\end{array}$ & 3 weeks & 4 \\
\hline Ritchie $1979^{\text {w33 }}$ & England & Tertiary care & $\begin{array}{l}\text { Clinical diagnosis } \\
\text { and investigations }\end{array}$ & $\begin{array}{l}\text { Patient reported improvement in global } \\
\text { symptoms (doctor required to agree) }\end{array}$ & 24 & $\begin{array}{l}\text { Hyoscine } 10 \mathrm{mg} \text { four times } \\
\text { daily }\end{array}$ & 3 months & 4 \\
\hline $\begin{array}{l}\text { D'Arienzo } \\
1980^{\text {w23 }}\end{array}$ & Italy & Tertiary care & $\begin{array}{l}\text { Clinical diagnosis } \\
\text { and investigations }\end{array}$ & $\begin{array}{l}\text { Patient reported improvement in global } \\
\text { symptoms }\end{array}$ & 28 & $\begin{array}{l}\text { Otilonium } 20 \mathrm{mg} \text { three times } \\
\text { daily }\end{array}$ & 4 weeks & 3 \\
\hline Fielding $1980^{\text {w20 }}$ & Ireland & Secondary care & $\begin{array}{l}\text { Clinical diagnosis } \\
\text { and investigations }\end{array}$ & $\begin{array}{l}\text { Physician reported improvement in global } \\
\text { symptoms }\end{array}$ & 60 & $\begin{array}{l}\text { Trimebutine } 200 \mathrm{mg} \text { three } \\
\text { times daily }\end{array}$ & 6 months & 3 \\
\hline Delmont $1981^{\text {w27 }}$ & France & Secondary care & $\begin{array}{l}\text { Clinical diagnosis } \\
\text { and investigations }\end{array}$ & $\begin{array}{l}\text { Physician reported improvement in global } \\
\text { symptoms }\end{array}$ & 60 & $\begin{array}{l}\text { Pinaverium (dose unclear) } \\
\text { one tablet three times daily }\end{array}$ & 30 days & 4 \\
\hline Page $1981^{\text {w21 }}$ & Unclear & $\begin{array}{l}\text { Primary and } \\
\text { secondary care }\end{array}$ & $\begin{array}{l}\text { Clinical diagnosis } \\
\text { and investigations }\end{array}$ & $\begin{array}{l}\text { Patient reported improvement in global } \\
\text { symptoms }\end{array}$ & 97 & $\begin{array}{l}\text { Dicycloverine (dicyclomine) } \\
40 \text { mg four times daily }\end{array}$ & 2 weeks & 4 \\
\hline Baldi $1983^{\text {w25 }}$ & Italy & Secondary care & $\begin{array}{l}\text { Clinical diagnosis } \\
\text { and investigations }\end{array}$ & $\begin{array}{l}\text { Decrease in patient reported abdominal } \\
\text { pain score }\end{array}$ & 30 & $\begin{array}{l}\text { Otilonium } 40 \mathrm{mg} \text { three times } \\
\text { daily }\end{array}$ & 4 weeks & 4 \\
\hline Nigam $1984^{\text {w35 }}$ & India & Secondary care & $\begin{array}{l}\text { Clinical diagnosis } \\
\text { and investigations }\end{array}$ & $\begin{array}{l}\text { Patient reported improvement in global } \\
\text { symptoms (doctor required to agree) }\end{array}$ & 42 & Hyoscine (dose unclear) & 12 weeks & 3 \\
\hline Ghidini $1986^{\text {w14 }}$ & Italy & Secondary care & $\begin{array}{l}\text { Clinical diagnosis } \\
\text { and investigations }\end{array}$ & Patient reported efficacy of treatment & 90 & $\begin{array}{l}\text { Trimebutine } 100 \mathrm{mg} \text { three } \\
\text { times daily or rociverine } \\
20 \mathrm{mg} \text { three times daily }\end{array}$ & 60 days & 3 \\
\hline Kruis $1986^{\text {w34 }}$ & Germany & Tertiary care & $\begin{array}{l}\text { Clinical diagnosis } \\
\text { and investigations }\end{array}$ & $\begin{array}{l}\text { Patient reported improvement in global } \\
\text { symptoms }\end{array}$ & 80 & $\begin{array}{l}\text { Mebeverine } 100 \mathrm{mg} \text { four } \\
\text { times daily }\end{array}$ & 16 weeks & 4 \\
\hline Virat $1987^{\text {w24 }}$ & France & Secondary care & $\begin{array}{l}\text { Clinical diagnosis } \\
\text { and investigations }\end{array}$ & $\begin{array}{l}\text { Patient reported improvement in global } \\
\text { symptoms }\end{array}$ & 78 & $\begin{array}{l}\text { Pinaverium } 50 \mathrm{mg} \text { three } \\
\text { times daily }\end{array}$ & 1 week & 2 \\
\hline $\begin{array}{l}\text { Centonze } \\
1988^{\text {w11 }}\end{array}$ & Italy & Tertiary care & $\begin{array}{l}\text { Clinical diagnosis } \\
\text { and investigations }\end{array}$ & $\begin{array}{l}\text { Patient reported improvement in global } \\
\text { symptoms }\end{array}$ & 48 & $\begin{array}{l}\text { Cimetropium } 50 \mathrm{mg} \text { three } \\
\text { times daily }\end{array}$ & 6 months & 4 \\
\hline Gilvarry $1989^{\text {w17 }}$ & Ireland & Secondary care & $\begin{array}{l}\text { Clinical diagnosis } \\
\text { and investigations }\end{array}$ & $\begin{array}{l}\text { Doctor reported improvement in global } \\
\text { symptoms }\end{array}$ & 24 & $\begin{array}{l}\text { Pirenzipine } 50 \mathrm{mg} \text { twice } \\
\text { daily }\end{array}$ & 4 weeks & 4 \\
\hline $\begin{array}{l}\text { Passaretti } \\
1989^{\text {w13 }}\end{array}$ & Italy & Tertiary care & $\begin{array}{l}\text { Clinical diagnosis } \\
\text { and investigations }\end{array}$ & $\begin{array}{l}\text { Patient reported improvement in global } \\
\text { symptoms }\end{array}$ & 40 & $\begin{array}{l}\text { Cimetropium } 50 \mathrm{mg} \text { three } \\
\text { times daily }\end{array}$ & 1 month & 4 \\
\hline Dobrilla $1990^{\text {w12 }}$ & Italy & Secondary care & $\begin{array}{l}\text { Clinical diagnosis } \\
\text { and investigations }\end{array}$ & $\begin{array}{l}\text { Patient reported improvement in global } \\
\text { symptoms }\end{array}$ & 70 & $\begin{array}{l}\text { Cimetropium } 50 \mathrm{mg} \text { three } \\
\text { times daily }\end{array}$ & 3 months & 4 \\
\hline Schafer $1990^{\text {w10 }}$ & Germany & $\begin{array}{l}\text { Primary and } \\
\text { secondary care }\end{array}$ & $\begin{array}{l}\text { Clinical diagnosis } \\
\text { and investigations }\end{array}$ & $\begin{array}{l}\text { Improvement in global symptoms } \\
\text { assessed by diary cards }\end{array}$ & 360 & $\begin{array}{l}\text { Hyoscine } 10 \mathrm{mg} \text { three times } \\
\text { daily }\end{array}$ & 4 weeks & 3 \\
\hline $\begin{array}{l}\text { Castiglione } \\
1991^{\text {w26 }}\end{array}$ & Italy & Tertiary care & Clinical diagnosis & $\begin{array}{l}\text { Improvement in global symptoms } \\
\text { (unclear whether patient reported) }\end{array}$ & 60 & $\begin{array}{l}\text { Otilonium (dose and } \\
\text { number of tablets unclear) }\end{array}$ & 1 month & 2 \\
\hline Pulpeiro $2000^{\text {w28 }}$ & Argentina & Tertiary care & $\begin{array}{l}\text { Clinical diagnosis } \\
\text { and investigations }\end{array}$ & $\begin{array}{l}\text { Patient reported improvement in global } \\
\text { symptoms }\end{array}$ & 75 & $\begin{array}{l}\text { Propinox (dose and number } \\
\text { of tablets unclear) }\end{array}$ & 4 weeks & 3 \\
\hline Glende $2002^{\text {w16 }}$ & Italy & $\begin{array}{l}\text { Secondary and } \\
\text { tertiary care }\end{array}$ & Rome I criteria & $\begin{array}{l}\text { Decrease in patient reported symptoms } \\
\text { (evaluated on a } 4 \text { point ordinal scale) of } \\
\text { one unit in at least one symptom for at } \\
\text { least two weeks }\end{array}$ & 317 & $\begin{array}{l}\text { Otilonium } 40 \text { mg three times } \\
\text { daily }\end{array}$ & 15 weeks & 3 \\
\hline Mitchell $2002^{\text {w18 }}$ & UK & $\begin{array}{l}\text { Secondary and } \\
\text { tertiary care }\end{array}$ & $\begin{array}{l}\text { Rome II criteria and } \\
\text { investigations }\end{array}$ & $\begin{array}{l}\text { Improvement in wellbeing from validated } \\
\text { diary cards }\end{array}$ & 107 & $\begin{array}{l}\text { Alverine } 120 \mathrm{mg} \text { three times } \\
\text { daily }\end{array}$ & 12 weeks & 5 \\
\hline
\end{tabular}




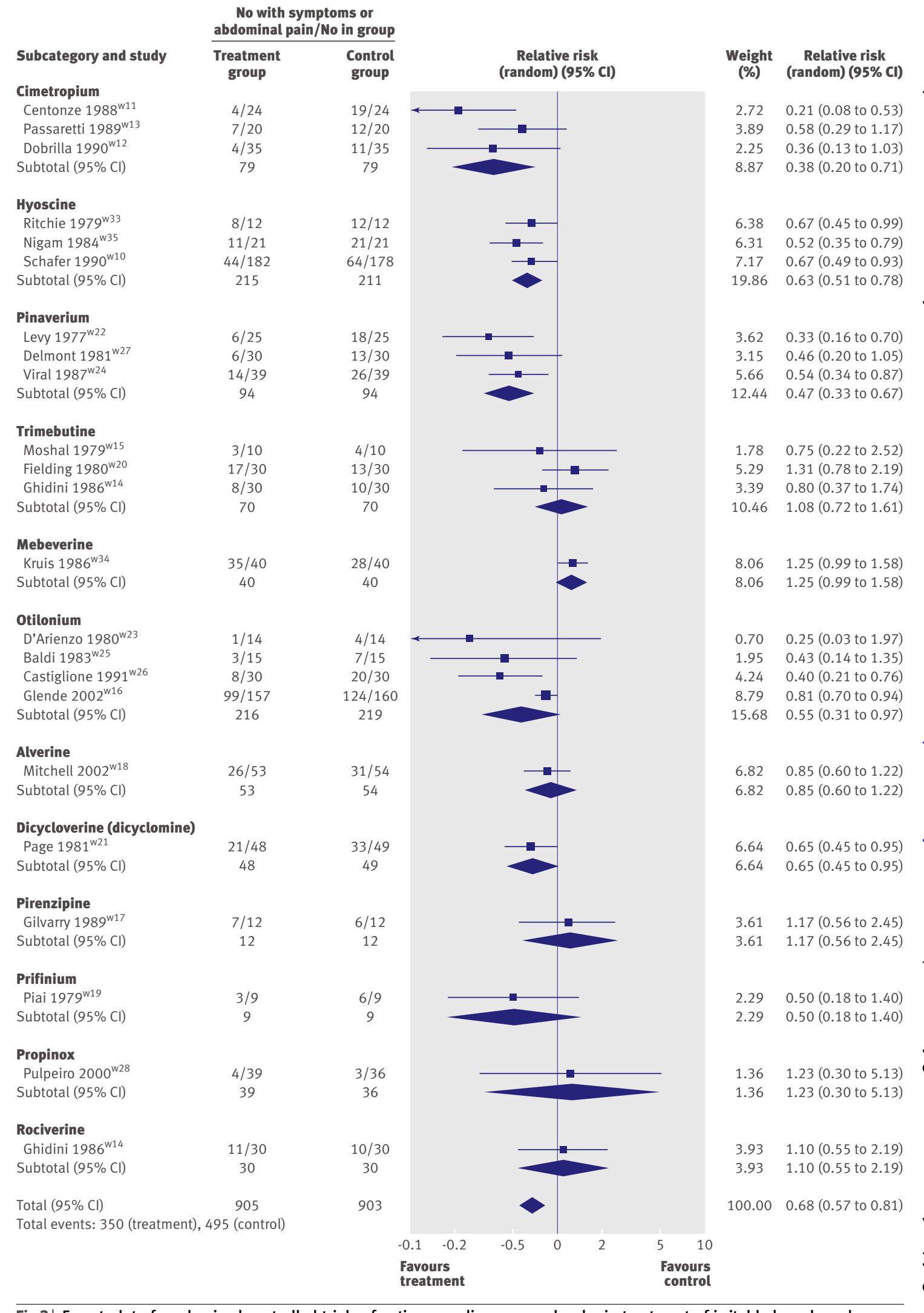

Fig 3 Forest plot of randomised controlled trials of antispasmodics versus placebo in treatment of irritable bowel syndrome. Events are number of patients with either global symptoms of irritable bowel syndrome or abdominal pain unimproved or persistent after treatment. See bmj.com for individual tests for heterogeneity and for overall effect 
significant (relative risk of persistent or unimproved symptoms $0.86,0.74$ to $1.01, \mathrm{P}=0.06$ ), with no statistically significant heterogeneity detected between studies $\left(\mathrm{I}^{2}=2.6 \%, \mathrm{P}=0.39\right)$.

\section{Adverse events}

Data on the total number of adverse events were provided by only four trials. ${ }^{\mathrm{w} 4 \mathrm{w} 6 \mathrm{w} 34 \mathrm{w} 35}$ These trials evaluated 251 patients, but as the number of adverse events was small the data were not pooled. A total of three patients receiving fibre reported adverse events compared with two in the placebo arms.

\section{Antispasmodics}

Twenty two studies compared 12 different antispasmodics with placebo in 1778 patients. ${ }^{\text {w10-w28 w33-w35 }}$ The proportion of women in each trial ranged from $39 \%$ to $83 \%$. Six studies reported on subtype of irritable bowel syndrome according to predominant stool pattern. ${ }^{\text {w11 } 12 \mathrm{w} 14 \mathrm{w} 15 \mathrm{w} 19 \mathrm{w} 24}$ One study recruited patients only with predominant constipation, ${ }^{\mathrm{w} 15}$ and in the remaining five studies between $22 \%$ and $64 \%$ of patients had predominant constipation. Table 2 shows the characteristics of the studies. None of the trials reported the method of allocation concealment.

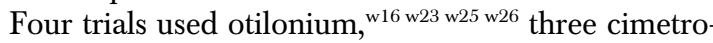
pium, ${ }^{\text {w11-w13 }}$ three hyoscine, ${ }^{\text {w10 }}{ }^{\text {w33 w35 }}$ three pinaver-

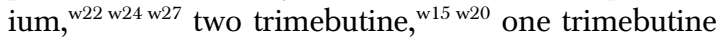
and rociverine, ${ }^{\mathrm{w} 14}$ and one each of alverine, ${ }^{\mathrm{w} 18}$ dicycloverine (dicyclomine), ${ }^{\text {w21 }}$ mebeverine, ${ }^{\text {w34 }}$ pirenzipine, ${ }^{\text {w17 }}$ prifinium, ${ }^{\text {w19 }}$ and propinox. ${ }^{\text {w28 }}$

In total, 350 of $905(39 \%)$ patients assigned to antispasmodics had persistent symptoms after treatment compared with 485 of $873(56 \%)$ allocated to placebo (relative risk $0.68,95 \%$ confidence interval 0.57 to 0.81 ), with statistically significant heterogeneity detected between studies $\left(\mathrm{I}^{2}=62.6 \%, \mathrm{P}<0.001\right.$; fig 3). The number needed to treat to prevent symptoms persisting in one patient was 5 (95\% confidence interval 4 to 9$)$. The Egger test suggested evidence of funnel plot asymmetry $(\mathrm{P}=0.03)$, but this seemed to be driven by a single small study; the Begg test did not confirm asymmetry $(\mathrm{P}=0.25)$. The treatment effect in favour of antispasmodics remained when only the 12 trials that scored 4 or more on the Jadad scale were considered in the analysis (relative risk of persistent symptoms 0.65 ,

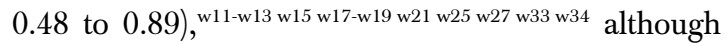
the heterogeneity observed between studies $\left(\mathrm{I}^{2}=70.2 \%\right.$, $\mathrm{P}=0.0001)$ persisted and there was evidence of publication bias (Egger test, $\mathrm{P}=0.007$ ).

The effect of different antispasmodics on symptoms of irritable bowel syndrome was examined separately. Four trials used otilonium in 435 patients. ${ }^{\text {w16 w23 w25 w26 }}$ Symptoms persisted in 111 of 216 (51\%) patients assigned to otilonium compared with 155 of $219(71 \%)$ of those receiving placebo (relative risk $0.55,0.31$ to $0.97, \mathrm{I}^{2}=59.5 \%$ ), and a number needed to treat of 4.5 (95\% confidence interval 3.0 to 10.0$)$. Three trials used cimetropium, ${ }^{\text {w11-w13 }}$ with 15 of 79 (19\%) patients randomised to cimetropium having persistent symptoms after treatment compared with 42 of $79(53 \%)$ receiving placebo (relative risk $0.38,0.20$ to 0.71 , $\left.\mathrm{I}^{2}=37.2 \%\right)$. The number needed to treat with cimetropium was 3.0 (2.0 to 12.5). Three studies randomised 426 patients to hyoscine or placebo. ${ }^{\text {w10 w33 w35 Symp- }}$ toms persisted in 63 of $215(29 \%)$ patients receiving hyoscine compared with 97 of $211(46 \%)$ allocated to placebo (relative risk $0.63,0.51$ to $0.78, \mathrm{I}^{2}=0 \%$ ), with a number needed to treat of 3.5 (2.0 to 25.0). Three trials used pinaverium in 188 patients. ${ }^{\text {w22 w24 w27 Symptoms }}$ persisted in 26 of $94(28 \%)$ patients assigned to pinaverium compared with 57 of $94(61 \%)$ receiving placebo (relative risk $0.47,0.33$ to $0.67, \mathrm{I}^{2}=0 \%$ ), and a number needed to treat of 3 (2 to 5). Three trials, totalling 140 patients, studied the effect of trimebutine. ${ }^{\text {w14 w15 w20 }}$ Twenty eight of $70(40 \%)$ patients assigned to trimebutine had persistent symptoms compared with 27 of $70(39 \%)$ allocated to placebo (relative risk $1.08,0.72$ to $\left.1.61, \mathrm{I}^{2}=0 \%\right)$. The relative risk of persistent symptoms in the single trials that used other antispasmodics were: mebeverine 1.25 (0.99 to 1.58), alverine 0.85 ( 0.60 to 1.22 ), dicycloverine 0.65 ( 0.45 to $0.95)$, pirenzipine 1.17 (0.56 to 2.45$)$, prifinium 0.50 (0.18 to 1.40$)$, propinox 1.23 (0.30 to 5.13$)$, and rociverine 1.10 (0.55 to 2.19$)$.

\section{Adverse events}

Thirteen studies reported a total number of adverse

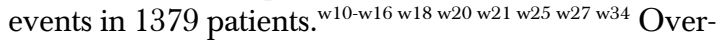
all, 101 of $704(14 \%)$ patients assigned to antispasmodics experienced adverse events compared with 62 of $675(9 \%)$ allocated to placebo. The

\begin{tabular}{|c|c|c|c|c|c|c|c|c|}
\hline Study & Country & Setting & $\begin{array}{l}\text { Diagnostic criteria for } \\
\text { irritable bowel syndrome }\end{array}$ & $\begin{array}{l}\text { Criteria to define symptom } \\
\text { improvement after therapy }\end{array}$ & $\begin{array}{l}\text { Sample } \\
\text { size }\end{array}$ & $\begin{array}{c}\text { Dose of } \\
\text { peppermint oil }\end{array}$ & $\begin{array}{l}\text { Duration of } \\
\text { therapy }\end{array}$ & Jadad score \\
\hline Lech $1988^{\text {w29 }}$ & Denmark & Secondary care & $\begin{array}{l}\text { Clinical diagnosis and } \\
\text { investigations }\end{array}$ & $\begin{array}{l}\text { Patient reported improvement in global } \\
\text { symptoms }\end{array}$ & 47 & $\begin{array}{l}200 \mathrm{mg} \text { three } \\
\text { times daily }\end{array}$ & 4 weeks & 3 \\
\hline Liu $1997^{\text {w30 }}$ & Taiwan & Secondary care & $\begin{array}{l}\text { Clinical diagnosis and } \\
\text { investigations }\end{array}$ & $\begin{array}{l}\text { Patient reported improvement in } \\
\text { abdominal pain }\end{array}$ & 110 & $\begin{array}{l}187 \text { mg three or } \\
\text { four times daily }\end{array}$ & 1 month & 4 \\
\hline $\begin{array}{l}\text { Capanni } \\
2005^{\text {w32 }}\end{array}$ & Italy & Secondary care & Rome II & $\begin{array}{l}\text { Improvement in global symptoms } \\
\text { assessed by validated questionnaire }\end{array}$ & 178 & $\begin{array}{l}2 \text { capsules three } \\
\text { times daily }\end{array}$ & 3 months & 5 \\
\hline $\begin{array}{l}\text { Cappello } \\
2007^{\text {w31 }}\end{array}$ & Italy & Secondary care & Rome II and investigations & $\begin{array}{l}\geq 50 \% \text { improvement from baseline in } \\
\text { overall irritable bowel syndrome symptom } \\
\text { score using questionnaire data }\end{array}$ & 57 & $\begin{array}{l}225 \mathrm{mg} \text { twice } \\
\text { daily }\end{array}$ & 4 weeks & 5 \\
\hline
\end{tabular}


commonest adverse events were dry mouth, dizziness, and blurred vision, but none of the trials reported any serious adverse events. The relative risk of experiencing adverse events with antispasmodics compared with placebo was 1.62 (95\% confidence interval 1.05 to 2.50 ), with statistically significant heterogeneity detected between studies $\left(\mathrm{I}^{2}=37.9 \%, \mathrm{P}=0.07\right)$, but no evidence of publication bias (Egger test, $\mathrm{P}=0.53$ ). The number needed to harm with antispasmodics was 17.5 (7.0 to 217.0$)$.

\section{Peppermint oil}

Four studies compared peppermint oil with placebo in 392 patients. $^{\text {w29-w32 }}$ The proportion of women in each trial ranged from $40 \%$ to $76 \%$. Only one study reported on disease subtype according to stool pattern and recruited $25 \%$ of patients with predominant constipation and $75 \%$ with predominant diarrhoea. ${ }^{\text {w31 }}$ Table 3 shows the characteristics of the studies. None of the trials reported the method of allocation concealment.

Fifty two of $197(26 \%)$ patients randomised to peppermint oil had persistent symptoms compared with 127 of $195(65 \%)$ receiving placebo (relative risk $0.43,0.32$ to 0.59 ; fig 4 ), with statistically significant heterogeneity detected between studies $\left(\mathrm{I}^{2}=31.1 \%\right.$, $\mathrm{P}=0.23)$. The number needed to treat with peppermint oil to prevent one patient having persistent symptoms was 2.5 (2.0 to 3.0). When only the three studies that scored 4 or more on the Jadad scale were considered in the analysis the relative risk of persistent symptoms was of a similar magnitude $(0.40,0.29$ to 0.55$)$, with no statistically significant heterogeneity detected between studies $\left(\mathrm{I}^{2}=22.0 \%, \mathrm{P}=0.28\right) .{ }^{\text {w30-w32 }}$

\section{Adverse events}

Only three studies reported data on adverse events, ${ }^{\text {w30- }}$ w32 and as these were few in number the data were not pooled. Five adverse events occurred among 174 patients assigned to peppermint oil compared with no adverse events in 171 patients receiving placebo.

\section{DISCUSSION}

This systematic review and meta-analysis has shown that fibre, antispasmodics, and peppermint oil are all more effective than placebo in the treatment of irritable bowel syndrome. The number needed to treat to prevent one patient having persistent symptoms was 11 for fibre, 5 for antispasmodics, and 2.5 for peppermint oil. Adverse events were significantly more frequent in those receiving antispasmodics than in those receiving placebo, but none of these was serious. As several different treatments were studied in the included randomised controlled trials, we carried out subgroup analyses.

When type of fibre was examined, wheat bran was no more effective at treating irritable bowel syndrome than placebo or a low fibre diet. The beneficial effect of fibre seemed to be limited to ispaghula husk, with a number needed to treat of 6 compared with placebo. However, significant heterogeneity was detected between trials. When only high quality studies were considered in the analysis this heterogeneity was diminished, but the difference in effect on symptoms in favour of ispaghula husk only reached marginal statistical significance.

Antispasmodics were of benefit, but again heterogeneity between study results was significant, and there was evidence of publication bias. Data were limited for many of the drugs licensed for use in the United Kingdom, such as mebeverine, dicycloverine, and alverine. It is difficult to know whether this is a true class effect of antispasmodics. Of all the drugs studied, most data were available for otilonium, trimebutine, cimetropium, hyoscine, and pinaverium. Trimebutine seemed to have no benefit over placebo in treating irritable bowel syndrome, whereas the other four drugs all significantly reduced the risk of persistent symptoms after treatment. Considerable heterogeneity was, however, detected between individual trials using otilonium and cimetropium and, although this was not the case when studies of pinaverium were pooled, the number of included patients was small. The best evidence for an individual compound seems to be for

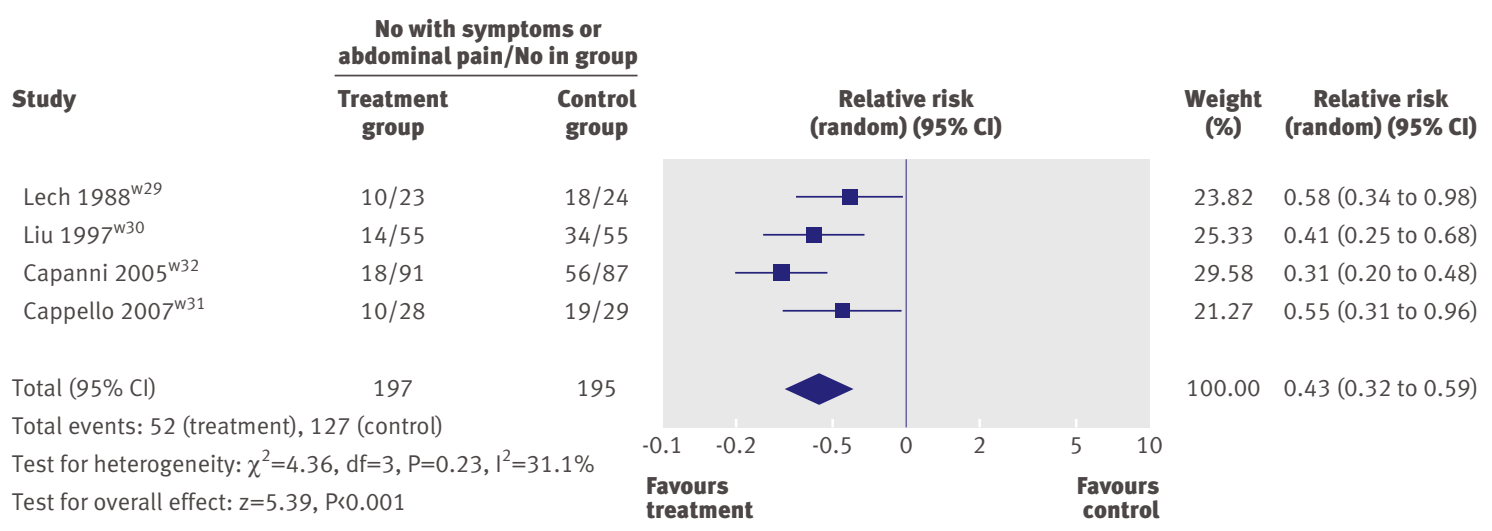

Fig 4 | Forest plot of randomised controlled trials of peppermint oil versus placebo in irritable bowel syndrome. Events are number of patients with either global symptoms of irritable bowel syndrome or abdominal pain unimproved or persistent after treatment 


\section{WHAT IS ALREADY KNOWN ON THIS TOPIC}

Irritable bowel syndrome is a chronic, relapsing and remitting disorder, which can be difficult to treat

Safe, effective treatments are required, as newer more expensive therapies have been withdrawn because of concerns about safety

Fibre, antispasmodics, and peppermint oil may fulfil this role, but evidence for their use is conflicting owing to methodological errors in previous systematic reviews

\section{WHAT THIS STUDY ADDS}

Fibre, antispasmodics (particularly hyoscine and otilonium), and peppermint oil were all more effective than placebo for treating irritable bowel syndrome

The numbers needed to treat with these therapies were 11,5 , and 2.5 , respectively

Doctors should consider ispaghula, antispasmodics (preferably hyoscine as first line treatment), and peppermint oil to treat irritable bowel syndrome

hyoscine, the efficacy of which was studied in over 400 patients. No statistically significant heterogeneity was detected, and 3.5 patients would need to be treated to prevent symptoms persisting in one patient. It would seem reasonable for general practitioners who want to begin a trial of antispasmodics to use hyoscine as first line treatment, but to consider other antispasmodics when this strategy fails.

Peppermint oil was also superior to placebo, although statistically significant heterogeneity was detected between study results, and only four randomised controlled trials were identified including fewer than 400 patients, so data were more limited than for fibre and antispasmodics. Three of these trials scored more than 4 on the Jadad scale, ${ }^{\text {w30-w32 }}$ but the treatment effect was similar when only these studies were included in the meta-analysis, and the heterogeneity observed between studies was no longer detected.

The reporting in this systematic review adheres to the quality of reporting of meta-analyses statement. ${ }^{29} \mathrm{We}$ have also specified the search strategy used, as well as our data extraction criteria. We believe that stating the criteria for data extraction should be standard in all systematic reviews that pool dichotomous data. The combination of our comprehensive search strategy and the translation of foreign language articles enabled us to identify studies with data from over 2500 people with irritable bowel syndrome.

Limitations of the current study arise from the quality of the studies eligible for inclusion, which in most cases was moderate to good, according to the Jadad scale. None of the included randomised controlled trials reported the method of allocation concealment, however, and as this has been shown to exaggerate treatment effect $^{30}$ the numbers needed to treat with these treatments may have been overestimated. Most trials were done before the Rome committee published their recommendations for the design of randomised controlled trials of therapies in functional gastrointestinal disorders. ${ }^{31}$ Only five of the included studies used the Rome criteria to define the presence of irritable bowel syndrome, ${ }^{\text {w1 }}$ w16 w18 w31 w32 although only nine were published after the first Rome classification was proposed in 1990, ${ }^{w 1}$ w4 w16 w18 w26 w28 w30-w32 and only two used a validated outcome measure to define improvement in symptoms after treatment. ${ }^{\text {w18 w32 }}$ However, many of the included trials met some of the other suggested methodological criteria, such as presence of double blinding and a minimum duration of therapy of 8 to 12 weeks. We preferentially extracted patient reported improvement in symptoms of irritable bowel syndrome or abdominal pain whenever trial reporting allowed this, which is also in line with these recommendations. Blinding of patients in these studies may not have been entirely successful owing to differences in consistency and texture between fibre and placebo, adverse events experienced with antispasmodics and, in the case of peppermint oil, the smell and taste of active treatment. The pooling of data from trials to give an overall treatment effect, and a number needed to treat, could be criticised by some as a result of differences in the methodology of individual included studies. We carried out sensitivity analyses to explore reasons for heterogeneity between studies and in all cases identified potential reasons for this, while still showing a significant treatment effect for most of the treatments we assessed.

Several previous systematic reviews have examined the role of these three treatments in irritable bowel syndrome. ${ }^{15-22}$ All of these, however, have limitations. Three reviews did not synthesise data, so no summary effect of individual treatments was reported. ${ }^{15-17}$ Of the five that extracted and pooled data in a meta-analysis all have numerous methodological errors, ${ }^{18-22}$ which render their findings potentially inaccurate. These include errors in the extraction of dichotomous data in a large proportion of included randomised controlled trials, ${ }^{18-22}$ inclusion of non-eligible studies (according to the investigators prespecified inclusion criteria) in four meta-analyses, ${ }^{18-2022}$ incorrect handling of data from cross over studies, ${ }^{18192122}$ failure to carry out an intention to treat analysis when trial reporting allowed, ${ }^{19-22}$ incorporating studies that included patients with other functional gastrointestinal diseases in the analysis, ${ }^{1820}$ and failure to identify eligible studies published at the time of the literature search, ${ }^{18-2022}$ leading to data on truly eligible patients being excluded from the analysis. These errors led to either an overestimation or underestimation of the pooled treatment effect in many of these meta-analyses. ${ }^{19-22}$ In addition, since these reviews were carried out further randomised controlled trials of all these treatments have been published.

Current guidelines for the management of irritable bowel syndrome are equivocal or conflicting in their recommendations for the use of these treatments, ${ }^{11} 122324$ but most of these have been informed by previous systematic reviews, which are potentially methodologically flawed for the reasons discussed, and this has implications for the statements made in them. In the UK, guidelines from both the National Institute for Health and Clinical Excellence and the British Society of Gastroenterology provide similar advice. ${ }^{11} 12$ Antispasmodics are recommended 
as first line treatment, particularly when pain and bloating are the predominant symptoms, although which of these drugs should be preferred is not stated. The use of insoluble fibre is discouraged because of concerns that it may exacerbate symptoms, an observation not borne out by our findings. Both organisations recommend that if fibre supplementation is required then this should be in the form of soluble fibres such as ispaghula. Finally, neither of these guidelines provides any statement on the role of peppermint oil in the management of irritable bowel syndrome.

The biological rationale for the efficacy of antispasmodics is unclear, but recent research using magnetic resonance imaging has shown that patients with irritable bowel syndrome and predominant diarrhoea have a reduced colon diameter as well as accelerated small bowel transit, ${ }^{32}$ so antispasmodics may act by reducing colonic contraction and transit time and therefore pain and stool frequency. Ispaghula husk may increase transit time in those with irritable bowel syndrome and predominant constipation. The efficacy of peppermint oil may arise from effects on smooth muscle, again reducing colonic contractility and pain owing to its calcium channel blocking activity. ${ }^{14}$ We were unable to examine the effect of different treatments according to predominant stool pattern reported by the patients, however, because few of the eligible trials reported these data as many predate the use of these subgroups, making it difficult to assign people to these categories retrospectively.

In summary, this systematic review and metaanalysis shows that ispaghula husk, antispasmodics (particularly hyoscine), and peppermint oil are all effective treatments for irritable bowel syndrome. Many of these are safe and available over the counter but, with the advent of newer more expensive drugs, are often overlooked as potentially effective treatments. Further large trials of these three agents in patients with irritable bowel syndrome, defined according to the Rome criteria, and using validated outcome measures are warranted. In the interim, current national guidelines for the management of the condition should be updated to include these data.

This study was done to inform the American College of Gastroenterology monograph on irritable bowel syndrome. We thank William Chey, Lawrence Brandt, Phillip Schoenfeld, and Edgar Achkar for their contributions to the discussion concerning the role of fibre, antispasmodics, and peppermint oil in the treatment of irritable bowel syndrome; and Premysl Bercik, Peter Bytzer, and Heidi Krall for assisting us with the translation of foreign language articles.

Contributors: All authors conceived and designed the study and drafted the manuscript and critically revised it for important intellectual content. ACF and PM acquired, analysed, and interpreted the data. ACF is guarantor.

Funding: This study was funded by the American College of Gastroenterology. The authors' work was independent of the funders. Competing interests: NJT has received consultancy fees from Procter and Gamble, Lexicon Genetics, Astellas Pharma US, Pharma Frontiers, Callisto Pharmaceuticals, AstraZeneca, Addex Pharma, Ferring Pharma, Salix, M GI Pharma, McNeil Consumer, Microbia, Dynogen, Conexus, Novartis, and Metabolic Pharmaceuticals, and has received research support from Novartis, Takeda, GlaxoSmithKline, Dynogen, and Tioga. EMMQ has received consultant's and speaker's bureau fees from Nycomed,
Boehringer Ingelheim, Procter and Gamble, Reckitt Benckiser, and Prometheus, and holds equity in Alimentary Health. PM holds a chair at McMaster University partly funded by an unrestricted donation by AstraZeneca, and has received consultant's and speaker's bureau fees from AstraZeneca, AxCan Pharma, Nycomed, and Johnson and Johnson. Ethical approval: Not required.

Provenance and peer review: Not commissioned; externally peer reviewed.

1 Longstreth GF, Thompson WG, Chey WD, Houghton LA, Mearin F, Spiller RC. Functional bowel disorders. Gastroenterology 2006;130:1480-91.

2 Agreus L, Talley NJ, Svardsudd K, Tibblin G, Jones MP. Identifying dyspepsia and irritable bowel syndrome: the value of pain or discomfort, and bowel habit descriptors. Scand J Gastroenterol 2000;35:142-51.

3 Hillila MT, Farkkila MA. Prevalence of irritable bowel syndrome according to different diagnostic criteria in a non-selected adult population. Aliment Pharmacol Ther 2004; 20:339-45.

4 Hungin APS, Whorwell PJ, Tack J, Mearin F. The prevalence, patterns and impact of irritable bowel syndrome: an international survey of 40 000 subjects. Aliment Pharmacol Ther 2003;17:643-50.

5 McKee DP, Quigley EM. Intestinal motility in irritable bowel syndrome: Is IBS a motility disorder? Part 1. Definition of IBS and colonic motility. Dig Dis Sci 1993;38:1761-2.

6 Aziz Q, Thompson DG, Ng VW, Hamdy S, Sarkar S, Brammer MJ, et al. Cortical processing of human somatic and visceral sensation. J Neurosci 2000;20:2657-63.

7 Trimble KC, Farouk R, Pryde A, Douglas S, Heading RC. Heightened visceral sensation in functional gastrointestinal disease is not sitespecific. Evidence for a generalized disorder of gut sensitivity. Dig Dis Sci 1995;40:1607-13.

8 Agreus L, Svardsudd K, Talley NJ, Jones MP, Tibblin G. Natural history of gastroesophageal reflux disease and functional abdominal disorders. Am J Gastroenterol 2001;96:2905-14.

9 Ford AC, Forman D, Bailey AG, Axon ATR, Moayyedi P. Irritable bowel syndrome: a 10-year natural history of symptoms, and factors that influence consultation behavior. Am J Gastroenterol 2008;103:1229-39.

10 Wilson S, Roberts L, Roalfe A, Bridge P, Singh S. Prevalence of irritable bowel syndrome: a community survey. Br J Gen Pract 2004;54:495-502

11 National Institute for Health and Clinical Excellence. Irritable bowel syndrome in adults: diagnosis and management of irritable bowel syndrome in primary care. 2008. www.nice.org.uk/nicemedia/pdf/ IBSFullGuideline.pdf.

12 Spiller R, Aziz Q, Creed FEA, Houghton L, Hungin P, Jones R, et al. Guidelines on the irritable bowel syndrome: mechanisms and practical management. Gut 2007;56:1770-98.

13 Harvey RF, Pomare EW, Heaton KW. Effects of increased dietary fibre on intestinal transit. Lancet 1973:301:1278-80.

14 Hills JM, Aaronson PI. The mechanism of action of peppermint oil on gastrointestinal smooth muscle. Gastroenterology 1991;101:55-65.

15 Jaliwala J, Imperiale TF, Kroenke K. Pharmacologic treatment of the irritable bowel syndrome: a systematic review of randomized, controlled trials. Ann Intern Med 2000;133:136-47.

16 Brandt LJ, Bjorkman D, Fennerty MB, Locke III GR, Olden K, Peterson W, et al. Systematic review on the management of irritable bowel syndrome in North America. Am J Gastroenterol 2002;97(suppl):S7-26.

17 Tack J, Fried M, Houghton LA, Spicak J, Fisher G. Systematic review: the efficacy of treatments for irritable bowel syndrome-a European perspective. Aliment Pharmacol Ther 2006;24:183-205.

18 Poynard T, Regimbeau C, Benhamou Y. Meta-analysis of smooth muscle relaxants in the treatment of irritable bowel syndrome. Aliment Pharmacol Ther 2001;15:355-61.

19 Lesbros-Pantoflickova D, Michetti P, Fried M, Beglinger C, Blum A. Meta-analysis: the treatment of irritable bowel syndrome. Aliment Pharmacol Ther 2004;20:1253-69.

20 Quartero AO, Meineche-Schmidt V, Muris J, Rubin G, de Wit N. Bulking agents, antispasmodic and antidepressant medication for the treatment of irritable bowel syndrome. Cochrane Database Syst Rev 2005;Apr 18;(2):CD003460.

21 Pittler MH, Ernst E. Peppermint oil for irritable bowel syndrome: a critical review and meta-analysis. Am J Gastroenterol 1998;93:1131-5.

22 Bijkerk CJ, Muris JWM, Knottnerus JA, Hoes AW, de Wit NJ. Systematic review: the role of different types of fibre in the treatment of irritable bowel syndrome. Aliment Pharmacol Ther 2004;19:245-51.

23 Drossman DA, Camilleri M, Mayer EA, Whitehead WE. AGA technical review on irritable bowel syndrome. Gastroenterology 2002;123:2108-31.

24 American College of Gastroenterology Functional Gastrointestinal Disorders Task Force. Evidence-based position statement on the 
management of irritable bowel syndrome in North America. Am J Gastroenterol 2002;97(suppl 2):S2-5.

25 Jadad AR, Moore RA, Carroll D, Jenkinson C, Reynolds DJ, Gavaghan DJ, et al. Assessing the quality of reports of randomized clinical trials: is blinding necessary? Control Clin Trials 1996;17:1-12.

26 DerSimonian R, Laird N. Meta-analysis in clinical trials. Control Clin Trials 1986;7:177-88.

27 Higgins JPT, Thompson SG, Deeks JJ, Altman DG. Measuring inconsistency in meta-analyses. BMJ 2003;327:557-60.

28 Egger M, Davey-Smith G, Schneider M, Minder C. Bias in metaanalysis detected by a simple, graphical test. BMJ 1997;315:629-34.

29 Moher D, Cook DJ, Eastwood S, Olkin I, Rennie D, Stroup DF. Improving the quality of reports of meta-analyses of randomised controlled trials: the QUOROM statement. Quality of reporting of meta-analyses. Lancet 1999;354:1896-900.

30 Juni P, Altman DG, Egger M. Assessing the quality of controlled clinical trials. BMJ 2001;323:42-6.

31 Veldhuyzen Van Zanten SJ, Talley NJ, Bytzer P, Klein KB, Whorwell PJ, Zinsmeister AR. Design of treatment trials for functional gastrointestinal disorders. Gut 1999;45(suppl 2):॥69-77.

32 Marciani L, Foley S, Hoad CL, Campbell E, Totman JJ, Cox E, et al. Accelerated small bowel transit and contracted transverse colon in diarrhoea-predominant irritable bowel syndrome (IBS-D): novel insights from magnetic resonance imaging (MRI). Gastroenterology 2007;132(suppl 1):A141.

Accepted: 24 September 2008 